\title{
Semiótica y Estética del Diseño
}

\begin{abstract}
Ante el relativismo y los aparentes desórdenes del "movedizo mapa del diseño" en la cultura postmoderna, proceder desde la academia universiraria a los estudios críticos de los objetos que el diseño pone en el escenario contemporáneo corresponde a una tarea que demanda ciertas formalidades que permitan esclarecer dos componentes esenciales: los estratos de sentidos y las estructuras portantes de dichos estratos. El presente arcículo expone las generalidades de un modelo para realizar esta labor bajo la óptica de la semiótica y estérica visual.
\end{abstract}

Palabras clave: diseño, serniótica, estética

\section{Circunscripciones}

El título de este trabajo se encuentra precedido por la palabra "semiótica", término que demanda algunas aclaraciones.

La primera de cllas tiene que ver con la línea ideológica a la cual se adscribe esta palabra. Comúnmente se Ie llama semiótica a los aspectos disciplinarios dedicados al estudio del signo, como también a toda la constelación de argumentos que orbitan en torno a estâ partícula fundamental, práctica que se lleva a efecto independientemente de cuales sean los parámetros disciplinares que se usan para hablar de ello.

El autor de este artículo cree que esto, además de una profunda equivocación, es metodológicamente errado, y las razones son las siguientes:

A finales del siglo $\mathrm{xIX}$ y las primeras décadas del $\mathrm{xx}$ surgieron dos vertientes, las cuales dieron origen a los estudios sistemáticos en torno al fenómeno llamado "signo", líneas ideológicas que se encuentran ligadas a los nombres de Ferdinand de Saussure y Charles Sanders Peirce, dos eruditos y académicos universitarios, el primero de Ginebra y el segundo de Cambridge, Massachusetts. 
Charles Peirce, hijo del matemático estadounidense Benjamin Peirce, se graduó en Química en la U. de Harvard (1863), recinto en el cual además fue profesor, como también enseñó lógica en la Universidad Johns Hopkins de 1879 a 1884.

Se suma a lo anterior sus estudios paralelos en filosofia, disciplina en la cual se le considera uno de los padres del Pragmatismo.

Ferdinand de Saussure perteneció a una familia de escritores y hombres de letras. Realizó sus estudios en las Universidades de Ginebra, Berlín y Leipzig, graduándose en esta última en el año de I880 con un trabajo sobre "El uso del genitivo absoluto en el sánscrito". En París, Saussure fue académico de gramárica comparada y en Ginebra enseñó lingüística general, lingüística indoeuropea y sánscrito.

El pensamiento de Peirce se encuentra fundado en la lógica unida a una estructura científica en el área matemática-algebraica. El cálculo clásico, cálculo deductivo, cálculo de relativos, cuantificadores, índices y representaciones gráficas, se combinan con las "máximas" pragmáticas y categorías peircianas en semiótica. Se suma a lo anterior uná lógica triádica asociada a la teoría de los modelos y la teoría matemática de las categorías.

La perspecciva de Saussure presenta notables diferencias. La tradición intelectual que se involucra con este sabio ginebrino es la lingüística, como sistema de rigor para comprender, entre otras cosas, el signo y ellenguaje humano. De su trabajo han surgido "linguísticas" específicas dedicadas a estudios involucrados con las ciencias sociales.

La semiología surgió de los trabajos de Saussure y hoy presenta un desarrollo tremendamente importante, ello es evidenciado no sólo en las publicaciones, sino también en los congresos que se celebran cada cierto riempo a lo largo de Occidente.

De todo lo anterior surgen diferencias conceptuales emanadas de las metodologías sobre las cuales se funda la estructura del signo para cada caso: una unión dual del significado y el significante para Saussure, y una relación triádica de medios, objetos e interpretantes para Peirce.

\section{Semiótica "peirciana"}

Los componentes que dan forma a la semiótica de tradición filosófica, específicamente a la línea fundada por Peirce en el último tercio del siglo $\mathrm{x} x \mathrm{x}$, la cual ha sido continuada en la actualidad por un número importante de investigadores en el tema, posee una seric de conceptos, ideas y argumentos con los cuales es posible estudiar de forma sistemática las imágenes producidas por la actividad del diseño:

Para que ello ocurra es necesario organizar dichos conscructos en un modelo que permita desarrollar el análisis de una forma coherente, y al mismo tiempo sea conducente a obtener productos de investigación. 
La finalidad de todo lo anterior es desplegar una metodología de estudio conducente al establecimiento de una construcción teórica, en donde los argumentos que la sustentan correspondan más bien a una razón crítica y no a un comentario caracterizado generalmente por un "buen escribir", el cual entre otras cosas pucde ocultar una falta de cultura visual y conceptual en torno al fenómeno del diseño.

Los actos de "lectura" de la imagen involucran por parte de un "lector competente" un acto que en la semiótica peirciana responde a la relación entre intérprete y los repercorios sígnicos que arquitecturan de forma específica una determinada imagen.

El problema desde esta perspectiva se presenta caso a caso. De acuerdo a cllo el sujeto con un cierto entrenamiento podrá construir argumentos críticos a partir de la lectura y descodificación de los conjuntos sígnicos dados en la geografia de la imagen.

Por su lado, el tejido figurativo de la imagen manifestado por lo interfigurala ${ }^{2} y$ lo intrafigural, a lo que se les suman los fenómenos cromáticos, tipográficos y morfológicos, mas las dimensiones semánticas y sintácticas ${ }^{3}$ dan forma a una construcción visual susceptible de ser interpretada.

Desde una rigurosa perspectiva, todo lo anterior demanda una responsabilidad semiótica y estética del sujeto "lector". Leer y descodificar en este caso no son lo mismo, aunque se puedan entender como "partes" de un mismo proceso. Descodificar supone un proceso de interpretación en donde la conciencia utiliza herramientas que no pertenecen a los repertorios frecuentes de nuestra vida ordinaria. Se trata de otras tipologías, de aquellas asociadas al mundo de las indagaciones que desarticulan las envolventes epidérmicas, ello con el fin de penetrar en el mundo fenoménico que no es aparente, y que subyace en los arcanos de la imagen.

El constructo que se obtiene mediante este procedimiento obedece a lo que se conoce como "argumento crítico", situación que emerge de un proceso reflexivo, el cual fue orientado por alguna metodologia debidamente validada.

Ejemplos de lo anterior se encuentran en los trabajos realizados de semiótica vinculada al diseño, ellos como una forma de estudios sistemáticos. Lo anterior no es un asunto reciente, ya Max Bense y Elizabeth Walther" (i975), a lo que sumaron otros seguidores de esta línea de pensamiento, desarrollaron investigaciones en esta área a partir de la segunda mitad del siglo $\mathrm{XX}$.

Entre todos ellos se deben mencionar "Signo y Diseño" (Bense,1973), "Descripciones semióticas en el ejemplo de tres aparatos telefónicos" (Kübler et al., 1972), labor que involucra al diseño industrial, y "Serniótica en la práctica del diseño"(Kübler,1976). 
Despejar los estratos de recubrimientos, es decir, la apariencia inmediata de una imagen, no es una tarea imposible, sí es una labor que requiere algunas condiciones por parte de quién efectúe dicho ejercicio. El acto de interpretar aquello que se despeja, tema de vital importancia para la semiótica visual, como también para la estérica de la recepción', demanda examinar las siguientes situaciones:

x. Entender una imagen, en este caso inserta en un objeto generado al interior de lo que se conoce como "diseño" es traducir por medio de un acto de interpretación los contenidos formales que yacen en el territorio de dicha imagen. Dichos contenidos se encuentran configurados por repertorios sígnicos, los cuales presentan la posibilidad de ser cualificados y cuantificados.

2. Considerar a la tipología de imagen expresada bajo los parámetros señalados en el párrafo anterior, es decir, como un todo compuesto por conjuntos de signos, los cuales a su vez forman un territorio semiótico que permite la interpretación, es bajo la óptica del autor de este arrículo, involucrar el pensamiento de Peirce y sus seguidores como Bense y Walther con la estérica del diseño. Lo anterior puede dar forma a una "semiótica analítica" orientada al fenómeno del diseño.

3. Desde la perspectiva del "lector" competente, es conveniente preguntarse sobre algunas situaciones de importancia, como por ejemplo cuáles son los códigos que maneja y el metalenguaje que usa, ambos como repertorios básicos para la interpretación. Dichos componentes juegan un papel de vital importancia en la construcción del argumento crítico que se espera de un investigador con formación académica.

De acuerdo a lo anterior, designo cuatro fases, las cuales caracterizan la acrividad de "lectura" de la imagen provista por el objeto de diseño:

La primera de ellas involucra el acercamiento al tejido figurativo dado en la construcción de la imagen. En esta primera etapa, el intérprete tiene como objetivo distinguir los conjuntos sígnicos que dan consistencia a la dualidad forma-función. El acercamiento al tejido figurativo demandará a quien realice esta labor un trabajo de descodificación de los elementos que estructuran la apariencia de la imagen, tema que debe seguir un orden preestablecido por un modelo.

La segunda fase corresponde a una evapa de cuantificación de los signos, labor que requiere una búsqueda y sistematización estético-semiótica en cada seccional que se ha determinado como muestra representativa. Está claro que en esta disposición los signos no funcionan por separado, ya que su acruación en conjunto da 
origen a la densidad de su estructura, sistema que puede ser develado si el operador logra efectuar una "lectura", cuya primera instancia será la de discriminar lo que en rigor constituye la anatomía de la obra: sus elementos formales como portadores de información, en otras palabras, conjuntos de signos.

Aquí cl discernimicnto ha jugado un papel fundamental, ya que el analista puede pasar desde niveles herméticos, enigmáticos y obscuros, al logro de una situación en donde la claridad de las ideas permite despejar el camino de la imagen estudiada.

Esta cuantificación de los signos, realizada a partir del reconocimiento sistemático de los diferentes componentes que determinan la extensión de la imagen, corresponde a una labor que efectúa el intérprete, en la cual los signos se estabilizan en la conciencia de este sujeto, actividad que escinde y al mismo tiempo objetiviza los repertorios. Ello se hace posible por medio de la cuantificación de las diferencias dadas entre las magnitudes signicas.

En esta operación que tipifica lo diverso dado en estas magnitudes, no se puede prescindir de los caracteres sensibles del objeto de diseño, ya que ellos constituyen las estructuras objetivas que permiten realizar la operación antes señalada, tarea que se efectúa gracias a la facultad otorgada por los saberes, para "pragnaatizar" el número involucrándolo al mismo tiempo con objetos de diseño.

Todo lo anterior dice relación con la búsqueda de un tipo de conocimiento sistemático cuyas características están dadas al interior de lo comprobable, medible y replicable, en este caso específico, se trata de todo aquello que tenga que ver con la indagación diagramática del tejido figurativo dado en la imagen, ello como un fenómeno observable y susceptible de medición.

La cualificación de los sistemas indexicálicos, icónicoso simbólicos dados en la extensión de la imagen corresponden a la tarea de la tercera fase. El testimonio obtenido por la exploración visual que tealiza el analista, unido a una dimensión semántica, permitirá establecer las correspondencias entre lo que Peirce, Bense y Walther designaron como ícono, índice y símbolo", ellos entendidos como clases de signos al interior del sisterna tricotómico de la seniótica filosófica.

Este acto de cualificar, como el de cuantificar componentes de una imagen, se encuentra inserto en un procedimiento muy cercano a lo que Max Bense? postuló como una semiótica-estética de la constatación. La obra de diseño considerada bajo estos aspectos se constituye como una identidad llena de información, es decir, como una especie de estado estético visual que es observable por medio de la determinación de clases de signos dados en la extensión de la imagen. 
Lo anterior también se encuentra enlazado con el pensamiento de Peirce, ello en cuanto a lo que significa realmente un estado de observación conducido por un sistema metodológico. En torno a ello, este filósofo norteamericano afirmó lo siguiente:

Una de escas diticulades proviene del hecho que los detalles se discinguen con menor facilidad y se olvidan antes que las circunstancias generales. Segun esea teoria. los rasyos gencrales existen en los detalles: de hecho, los detalles son coda ha imagen. En consecucneia, parece mery extrano que aquello que solo existe en forma secundaria en la inagen cause más impresion que la imagen misma.

Más adelante agrega:

pero el argumento conclayente, contra ha afimación de que renemos inzágeneso represcnaciones absolutanente deterninadas por la percepcion. reside en que en tal caso cenemos en cada una de estas representaciones los materiales para una dosis infinica de cognición consciente, de io cual. sin cmbargo, casinunca nos enreramos ${ }^{3}$.

Por su lado, Max Bense cuando examinó fenómenos visuales los vinculó con aspectos vitales de la semiótica de la percepción. Ello llevó sus concepciones de los signos relacionados con el objeto hasta "los hechos fundamentales de la percepción como signos fundamentales de la semiótica, tanto estética como teoréticamente" ${ }^{9}$.

La arquitectónica del sistema inserto en el objeto de diseño, a estas alturas de su discernimiento, se presenta ante la mente del analista, menos confuso, su apariencia, que pudo ser engañosa a primera vista, se torna hacia los esrados de clarificación sígnica.

La cuarta fase corresponde a la formulación teórica emanada del proceso anterior, es decir, a la constitución de un argumento enlazado con la crírica. Esta última palabra entendida bajo los parámetros que Emmanuel Kant asignó a este término: "el tribunnal que garantice a la razón en sus pretensiones legítimas, pero que condene a las que no tienen fundamento" to.

Lo primero que se debe diferenciar en esta sección es declarar que la crítica del diseño, además de escasa, se encuentra en diferentes niveles. Por un lado se pueden observar aquellos ejercicios orales o escriturales que están dirigidos a un público común y cuya labor puede ser comunicacional y quizás en algunos casos, orientadora. En el otro extremo se sitúa aquella crítica de carácter epistémico, cuya finalidad es el discernimiento penetrante, el cual debe ser acompañado por un manejo riguroso de los metalenguajes que comprende la disciplina a la cual se refiere. El sentido de este artículo se sirúa en esta segunda aclaración.

La formulación teórica señalada en el párrafo anterior corresponde a la puesta en escena de todo aquello que se desprendió del 
estudio realizado en los pasos anteriores.

Esta "formulación teórica" como una resultante de un proceso crítico debe contener algunas evidencias como:

a. El advenimiento de un "logos semántico" entendido como un constructo de verdad proposicional, el cual emergió de la experiencia semiótica y estética con la obra de diseño.

b. Una relación entre pensamiento crítico, obra de diseño y lenguaje oral-escritural, relación que permitió el estudio claantitativo y cualitativo de los signos dados en la extensión de la imagen.

c. Una semiosis estética conducente a la instauración de conclusiones cuyos contenidos aporten nuevos saberes en torno al fenómeno estudiado.

\section{Modelo y modelización: generalidades de una propuesta}

La formalización semiótica y estética inscrita en un modelo para escudriñar la imagen, en este caso adscrita a las producciones del diseño, tiene por finalidad desarrollar una metodología de estudio conducente al establecimiento de una construcción teórica, lugar en donde los argumentos que la sustentan correspondan más bien a una razón crítica y no a un comentario caracterizado generalmente por un discurso de carácter epidérmico.

En materias atingentes a las imágenes, el acto de formalizar presupone un procedimiento por el cual se edifica un determinado sistema o conjunto, el cual es gobernado por algunas coherencias asociadas a modelos reales.

En este caso se reconocen dos situaciones: la tipología de objeto como producto del diseño, y la construcción teórica que se vincula con lo anterior.

Las reglas operativas de formación son establecidas por el intérprete capacitado para dicha labor, del cual se espera rigor y Ienguaje apropiado en cuanto a su profundidad y especificidad en la edificación de su discurso. La doctrina Peirce-Bense designa esta situación con el nombre de "interpretante lógico" :r.

De acuerdo a lo señalado con antelación, la tarea primordial de este intérprete serála de aclarar las condiciones de existencia del "programa iconográfico" de la imagen, programa que se encuentra compuesto por varias situaciones manifestadas por medio de signos visuales.

Las palabras "programa iconográfico" " deben entenderse como la resolución estructural dada al interior del perímetro de la obra. En esta área se organizan todas las categorias sígnicas que nacen a partir de las primeras intervenciones sobre el soporte escogido por el diseñador. De estos trabajos iniciales, asunto que puede designarse como un "estado 
embrionario", hasta las diferentes posibilidades de finalización de la obra, existen fases que van orientando la configuración del programa.

Una de las caracteristicas que he asignado al programa iconogrático de la imagen, tal como lo adverrí en uno de los párrafos iniciales de este artículo, es reconocerlo como un gran conjunto de signos, cuyos diferentes emplazamientos, magnitudes y naturalezas obedecen a principios que pueden ser develados mediante un proceso de formalización semiózica, es decir, desde las perspectivas señaladas con antelación; el programa iconográfico se muestra como un campo cuya geografía puede ser abordada por las matrices teóricas propuestas por el ámbito disciplinar de la semiótica.

La anatomía del eikón (imagen) procesada por el proceso de formalización antes señalado, en términos generales, contiene los siguientes pasos:

Una primera parte orientada a la determinación del programa iconográfico, sección en la cual existen cuatro componentes, cuyas rúbricas se encuentran dadas por las palabras "identidad estructural", ellas van del $\mathrm{r}$ al $\mathrm{r} v$, y todo este conjunto da forma a lo que he designado como "lo constructivo sintáctico", es decir, y siguiendo a Morris" (1994), el propósito es desentranar las leyes de formación del fenómeno inserto en la imagen provista por el objeto de diseño, ello en cuanto a como se encuentran dadas las evidencias formales a través de los repertorios sígnicos.

Los sistemas codificados y volumetrías (diseño $3 \mathrm{D}$ ), el tratamiento de los "morfolegisignos", la configuración del código espacial y las cromatologías con sus respectivas condicionantes de recepción, pretenden dar cuenta de todos aquellos detalles que según Peirce, además de ser los portadores de los rasgos generales, y de que los detalles "son toda la imagen", a su vez permiren que esa "dosis cognición consciente" I+ ocurra con un fin: el de enterarse de la lógica constructiva de una obra de diseño.

El segundo seccional del modelo se encuentra articulado por dos grandes aspectos: las categorías y aspectos de los perceptemas y la formulación de la transferencia y el intlujo.

Max Bense introdujo el concepto de "perceptema" is para dererminar las relaciones forma-color en el mundo de la percepción visual. A partir de las precisiones que realizó en su trabajo, he agregado el principio de "condensación", el cual se encuentra referido a cómo la relación anterior dada en una imagen, encierra las intenciones de un autor, ellas vertidas en procesos de representación (símbolos), o de reproducción (íconos). Lo anterior tiene que ver con el acto de producir un "enunciado de verdad proposicional", es decir, el analista debe articular un argumento basado en las mediciones tealizadas con 
antelación, asunto que ya advertí en párrafos anteriores.

El tercer y último seccional se encuentra orientado a la deterninación de isomorfías entre programas iconográficos, es decir, cómo y en qué estado se encuentra la obra de diseño seleccionada para el estudio, ello en relación a otros trabajos que se produjeron en diferentes latitudes.

La idea expresada en las palabras "isomorfías entre programas iconográficos" apunta directamente a un tópico en donde las correspondencias entre sistemas de discños puedan ser demostradas con evidencias, las cuales a veces presentan algunas dificultades en su detección.

A la fecha, cste modelo que he expuesto en forma resumida ha sido aplicado desde el año 2005, en los cursos de Semiótica de la Imagen ry rr en la carrera de Discño, adscrita a la Faculcad de Arquitectura y Urbanismo de la Universidad de Chile.

Dicho modelo tienc su origen en otros sistemas de la misma línea ideológica los cuales diseñé para varios proyectos Fondecyt relacionados con el fenómeno de la pintura, escultura e historia del arte en Chile, trabajos que se originaron en 1997 , en los cuales el autor de este artículo, como primer coinvestigador inserto en un equipo de trabajo interuniversidades, tuvo la responsabilidad de "semiotizar" los análisis para cada caso. Se trata de cuatro proyectos ya concluidos, el último entregado su informe final en marzo de zorr. En la actualidad, este equipo se ha adjudicado un quinto proyecto rotulado con el nombre de "Construcción del gusto: la crítica de arte en Chile desde 1849 az970", en el cual actúa como investigador responsable el $\mathrm{Dr}$. Pedro Zamorano de la Universidad de Talca, y como segundo coinvestigador el Dr. Alberto Madrid de la Universidad de Playa Ancha.

\section{Notas}

1. La reunión diseño-diseñador genera diversas categorías de objetos bi y tridimensionales, los cuales además de su intencionalidad determinada por la relación forma-función son portadores de una imagen. la cual presenta modalidades en su dimensión sintáctica y seméntica, es decir, existen ordenamientos sígnicos en donde hay códigos de organización. como también lo anterior kiene la misión de ser portadores ce sentido.

2. Estos dos conceptos fueron introducidos por Jean Blaize Grize en "Observaciones soire la estructura de la geometria elemental", trabajo que corresponde al capítulo Hl del libro "La epistemoiogia del espacio", obra en la cual Jean Piaget es el autor principal. He tomado esios términos y los he conducido hacia las dimensiones de la imagen, to cual constituye un camino diferente de lo que Blaise Grize consideró como "geometria elemental". La imagen inserta en los objelos gue produce el diseño gráfico posee otros componentes organizados por los códigos del color, la forma, el espacio, las tipografias, etc. Todos ellos se cruzan dando forma a un tejido figgusativo el cual puede ser de alta complejicfad. 
3. Las dimensiones aqui señaladas se encuentran basadas en io que Chartes Morris postuló para ellas en su obra "Fundamento de la teoría de los signos", originalmente en lengua inglesa cuya data es de 1938. La editorial Paidos-Comenicación publicó para los lectores de lengua hispana dos ediciones de esta obra, la segunda as de 1994.

4. A partir de 1960. Max Bense y Elizabeth Walther, entre muchos otros europeos, se constituyeron en los eruditos más importantes de la semiótica y estética derivada del pensamiento de Charles Sanders Peirce.

Max Bense fue un ciendífico (Or. en física) y un fitósón dedicado a la estética. Por su lado, Elizabeth Walker, erudita en romanistica, germanistica y lisica, la cual obtuvo su doctorado en 1959 con la tesis "El rol de la lógica de Port Royal en los inicios de la historia de las ciencias exactas", colaboró con Bense en la dílusión y desarrollo del persarniento "peirciano" en Alemania, especialmente en la Technische Hochschule de Suttgar. En sus trabajos existen obras dedicadas a la semiótica del diseño y la arquitectura. Entre todas las obras que produjeron en coniunto, se destaca aquella lituada "La semiótica, guía alfabética", texto-diccionario que contiene doscientos noventa y dos términos adscritos a la semiótica perteneciente a la linea fundada por Peirce. Muchas de estas palabras, que constituyen el metalenguaje de especialicad, dejan ver el severo respeto por el filosofo nortearnericano, ello se aprecia en las diferentes definiciones, ya que al inicio de ellas los autores antes señalados comienzan diciendo: "según Peirce", o "si se concibe con Peirce", o "junto a Peirce".

5. Me retiero con "estética de la recepción" a la línea de pensamiento generada en la Universidad de Konstanz en Alemania. En esta casa de estudios superiores Hans Robert Jauss y Wolgang iser, como los principales protagonistas, desarrolaron a partir de los años sesenta del siglo pasado una corriente de critica literaria y pensamiento estético en donde el papel del sujeto lector-intérprete juega un papel primordia!. Con ellos participaron tiltosofos de la envergadura como Hans Georo Gadamer, maestro de la hermenéutica del siglo XX. Rainer Warning publico en 1979 la obra "Rezephonsästhetiti", obra que la edilorial Visor en 1989 presentó en castellano para los Jectores de habla hispana con el nombre de "Estética de la recepción", libro de especiał interés para quienes aspirann a conocer en profundidad esta forma de comprender un texto. Dicha publicación contiene el pensaniento de los principales exponentes de esta línea ideológica, tałes como Román Ingarden, Fêlix Vodicka, Michael Riftaterfe, Stanley Fisch, además de los ya antes señalados, Iser y Jauss.

6. Los autores aqui señalados delinieron al icono, el símbolo y al indice como clases de signos. Peirce dio inicio a esta concepción la cual se observa en varias secciones de sus "Collected Papers". Bense y Walther en su "La senniótica, Guia Alfabética" a partir de la construcción peirciana desarrollaron estos criterios. El signo como una relación triadica entre medios. objetos e interpretantes posee una estructura diferente a la concepcion dual más conocida que provee la linguisistica, que es la unión de signilicado y signilicante. En la triada Peirce-Bense, el segundo contonente corresponde a la relación signo-objeto, la cual se divide a su vez en iconos, indices y simbolos. Cada uno de estos componentes posee deteniciones y alcances especilicos. La semiotica de tradición tilosófica se deline, entre ofras cosas, por estas precisiones en torno a las clases de signos.

7. No es extraño que Bense haya desarroliado este tipo de ideas, sus códigos formalivos entregados por sus estudios superiores en Matemáticas. Física y Filosofía en las Universidades de Köln, Basilea y Bonn, en esta úllima se doctoro con una tesis sobre Mecánica Cuântica y relativitad (1937), le conducen a esta clase de formulaciones. Bense es uno de los teóricos en semiblica y estética cuyos origenes se encontraron en una primera 
instancia en el neopositivismo, su fin fue indagar los tenómenos del arte, el diseño, la arquitectura, la cibernética y la comunicación. En su quehacer intelecchal se aprecian rasgos importantes provenientes de Charles S. Peirce, Charles Morris, Georges Birkhoft y Christián von Ehrenfels

8. Los estractos cilados aparecen en "Obra Lógico-Semística" de Ch. S. Peirce, pág. 81, en el seccional rotulado como "Algunas consecuencias de las cuatro incapacidades (referencia original: Journal of Speculative Piilosophy, pp. 140-157, vol. 29).

9. Más información puede encontrarse en la página 119, de "La semiótica, guía alfabética"

10. N. Abbagnano, en su Diccionario de filosofía (página 263) desarrolla más extensamente los criterios en tomo la palabra "critica", y la involucra con otros autores como Locke y Hegel.

11. La semiótica derivada de Peirce entiende como "interpretante lógico", "al efecto significativo de un signo que es un pensamiento o un signo mental" (p.90-La semiótica, guia allabética). Elizabeth Walther en su libro "Teoria de los signos" desarrolto la teoria de los interpretantes (pp. 105-106), la autora afirma:

"El interpretante final (interpretante normado, úllimo o efectivo de acuerdo a las denominaciones que Peirce ha empleado). es el autentico gentino interpretante que Peirce divide en: 1. Salurante o generador de placer; 2, práctico o generador de acciones, y 3 . pragmático o generador de autocontrol, que puede cienominarse tazmbiên generador de reflexiones". Más adelante la autora agrega: "Tal vez podríamos nombrar en este luggar la tricotomía que, un paso ulterior, Peirce denomina: 1. Interpretante emocional; 2. Interpretante energético, y 3 . Interpretante lógico, que corresponde casi a las explicaciones de este interpretante final.

12. Las palabras "programa iconogrático" de la imagen fueron desarroiladas teớricamente para un modelo de análisis semítico de la pintusa de caballete y mural. Este trabajo perteneció al proyecto Fondecyt 41040858 "Asecio exógeno a la pintura chilena, 1920-1960". El contenido de este trabajo tue publicado en actas de Congresos de Semiótica realizados en Chile y Argentina entre 2004 y 2006

13. Más información sobre estas consideraciones pueden encontrarse en la obra de Charles Morris.

14. Se debe recordar la cita de Peirce seialalada en el pie de página n ${ }^{2} 8$.

15. Las definiciones y los componentes de un perceptema plecien ser consullados en "La serniótica, gúa affabética", pp. 29 -30.

\section{Bibliografía}

Abbagnano, Nicola, Diccionario de filosotia, 1963. Fondo de Cultura Econónica, Mexico.

Bense, Max: Walther. Elizaherl 3. La scmiórica, guía allabérica, 1975. Anagrama. Barcelona.

Morris, Charles, Fundamento de la teoria de los signos, 1994. P’aidos Comunicación. Barcelona.

Peirce. Clarles. Obra Logico-Semiótica, 198\%. Taurus Comunicacionn. Madrid.

Piaget. Jcan. La epistemología del espacio, 1972. El Arenco, Bucnos Aires.

Walther. Elisabcth. Teoria de los signos, 1994. Dolmen estudio-Ediciones pedagógicas chilcnas S.A., Santiago.

Warning. Raincr. Estérica de la recepcion, 19\&9. Visor. La barca de la Medusa. Madrid. 